\title{
Searching for cool core clusters at high redshift
}

\author{
J. S. Santos ${ }^{1}$, P. Rosati ${ }^{2}$, P. Tozzi ${ }^{3}$, H. Böhringer ${ }^{1}$, S. Ettori ${ }^{4}$, and A. Bignamini ${ }^{3}$ \\ 1 Max-Planck-Institut für extraterrestrische Physik, Giessenbachstraße, 85748 Garching, Germany \\ e-mail: jsantos@mpe.mpg.de \\ 2 European Southern Observatory, Karl-Schwarzchild Strasse 2, 85748 Garching, Germany \\ 3 INAF - Osservatorio Astronomico di Trieste, via G.B. Tiepolo 11, 34131 Trieste, Italy \\ 4 INAF - Osservatorio Astronomico di Bologna, via Ranzani 1, 40127 Bologna, Italy
}

Received 9 October 2007 / Accepted 8 February 2008

\section{ABSTRACT}

\begin{abstract}
Aims. We investigate the detection of Cool Cores (CCs) in the distant galaxy cluster population with the purpose of measuring the $\mathrm{CC}$ fraction out to redshift $0.7 \leq z<1.4$. Using a sample of nearby clusters spanning a wide range of morphologies, we define criteria to characterize cool cores, which are applicable to the high-redshift sample.

Methods. We analyzed azimuthally-averaged surface brightness (SB) profiles with the known scaling relations, and we fitted single/double $\beta$ models to the data. Additionally, we measured a surface brightness concentration, $c_{\mathrm{SB}}$, as the ratio of the peak over the ambient SB. To verify that this is an unbiased parameter as a function of redshift, we developed a model independent "cloning" technique to simulate the nearby clusters as they would appear at the same redshifts and luminosities as those in the distant sample. This method is based on the application of the cosmological surface brightness dimming to high-resolution Chandra images, assuming no intrinsic cluster evolution. We obtained a more physical parameterization of the CC presence by computing the cooling time at a radius of $20 \mathrm{kpc}$ from the cluster center.

Results. The distribution of the SB concentration and the stacked radial profiles of the low- $z$ sample, combined with published information on the CC properties of these clusters, show 3 degrees of SB cuspiness: non-CC, moderate, and strong CC. The same analysis applied to the high- $z$ clusters reveals two regimes: non-CC and moderate $\mathrm{CC}$. The cooling time distribution corroborates this result by showing a strong negative correlation with $c_{\mathrm{SB}}$.

Conclusions. Our analysis indicates a significant fraction of distant clusters harboring a moderate $\mathrm{CC}$ out to $z=1.4$, similar to those found in the local sample. The absence of strong cooling is likely linked with a higher merger rate expected at redshift $z>0.7$, and should also be related to the shorter age of distant clusters, implying less time to develop a cool core.
\end{abstract}

Key words. X-rays: galaxies: clusters - galaxies: clusters: general - galaxies: cooling flows - galaxies: high-redshift

\section{Introduction}

Galaxy clusters are filled with hot, diffuse gas - the intracluster medium (ICM) - which strongly emits in X-rays. While the cluster dark matter distribution is generally described by its gravitational potential (Navarro et al. 1997), the physical processes that govern the behavior of the ICM are complex and include non gravitational phenomena. Cool cores are a manifestation of these intricate processes.

According to observations (Jones \& Forman 1984; Fabian et al. 1994; Peres et al. 1998; Chen et al. 2007), relaxed galaxy clusters are most often found to exhibit at their centers a significant drop in the ICM gas temperature due to radiative cooling. It is by now established that the cooling time in the central regions of clusters is shorter than the Hubble time, thus originating very dense cores. Therefore, we expect to observe a surface brightness (SB) peak in these regions (with a typical radius of the order of $70 \mathrm{kpc}$ - see, for example, Vikhlinin et al. 2005) along with some other features such as an enhanced iron abundance.

The well-known cooling flow problem stems from the observation that the few detected emission lines are not as strong as expected to justify the predicted cooling rate. Further observational evidence is found in the ratio of the central temperature with respect to the global cluster temperature $\left(T_{\text {central }} / T\right)$, which remains at a factor $\sim 1 / 3$ (Peterson et al. 2003;
Bauer et al. 2005), and the mass deposition rates $(\dot{M})$ are much smaller than expected (Edge \& Frayer 2003).

The existence of a feedback mechanism that counteracts the cooling is now widely accepted and currently, the most debated picture is heating by active galactic nuclei (AGN) (see review by Fabian et al. 1994). Although several plausible models exist that may explain how this mechanism works (Churazov et al. 2000; Brüggen \& Kaiser 2002), and there is evidence for enough output mechanical energy to suppress cooling, it remains unclear how this energy is distributed in a homogenous way, such that cool cores appear in the form observed. Nevertheless, the cool core - AGN connection has been unambiguously demonstrated with observations, e.g., Burns (1990), Eilek (2003), Sanderson et al. (2006), report that nearly every cooling flow cluster hosts a radio-emitting AGN, creating cavities in the X-ray gas.

There is clear evidence for clusters harboring cool cores, however many clusters do not show any signatures of cooling. At this point, it may be pertinent to question whether there is a $\mathrm{CC} /$ non-CC bimodal cluster population. In this the case, are $\mathrm{CC}$ clusters primordial and were some of them (the non-cool core clusters) wiped out by mergers or an AGN heating overshoot? Conversely, some authors support a scenario in which non-CC evolve to CC clusters if no major mergers occur (O'Hara et al. 2006). 
The role of mergers in destroying cool cores has long been debated (Fabian et al. 1984), both from observational results and predictions from simulations. Currently, observations seem to favor CC destruction through cluster mergers (Allen et al. 2001; Sanderson et al. 2006). Simulations, however, yield ambivalent results. Using adaptive mesh refinement simulations, Burns et al. (2008) advocate that non-cool core clusters undergo major mergers that destroy embryonic cool cores while CC clusters grow at a slower rate without suffering early substantial mergers. However, an analysis of smoothed particle hydrodynamical simulations of X-ray cluster mergers by Poole et al. (2006), suggests that the heating of the ICM arising from mergers is not sufficient to prevent cooling. They argue that, in the $\Lambda C D M$ scenario, the merger rate is too small to account for the local abundance of non-CC clusters whose disruptions occurs on short timescales. We note, however, that a good agreement in the overall properties of observed and simulated $\mathrm{CCs} / \mathrm{NCCs}$ is found by Burns et al., who were able to qualitatively reproduce the temperature structure observed in cool cores.

The present observational census on the abundance of cool core clusters sets a fraction of Cool Core Clusters (CCC) in the local Universe in the range of 50-70\% (Peres et al. 1998; Chen et al. 2007). As we move to increasing redshifts, there are fewer results on this topic. Using spatially resolved spectroscopy, Bauer et al. (2005) presented a comparative study of cool cores at low- and intermediate-redshift (mean $z=0.06$ and 0.22 , respectively). The cool cores at intermediate redshift show the same temperature decrement, $T / T_{\text {central }} \sim 3-4$, as the nearby CC's, and have a frequency rate similar to the local one. The central cooling time measurements confirmed this result. Consequently, they find no signs of evolution of the cool core fraction in the redshift range $z \sim 0.15-0.4$ to now.

At present, the knowledge of the high redshift cluster population is still rather poor: only $7 \mathrm{X}$-ray selected clusters with $z>1$ have been confirmed (Rosati et al. 1999; Perlman et al. 2002; Stanford et al. 2002; Mullis et al. 2005; Stanford et al. 2006). In particular, determining and quantifying the existence of cool cores at high redshift remains largely unexplored due to the lack of photon collecting power of current instruments to provide spectra with sufficient statistics for distant CC's. A first attempt to characterize a peaked core in high-redshift systems was undertaken in Ettori et al. (2004), using double beta model fitting. The outcome of this analysis states that a second beta model component cannot be required to describe the distant cluster data - at least, not in the same way as observed for nearby cool core clusters. Recently, Vikhlinin et al. (2006) presented a study on the evolution of CCC at $z>0.5$ based on pure imaging data, using a distant cluster sample drawn from the 400SD survey imaged with Chandra. These authors claim a strong evolution of the $\mathrm{CC}$ fraction in this redshift range, implying that we are missing cooling flows in the distant cluster population. However, this claim has been recently challenged with simulations by Burns et al. (2008), who found no evidence for such a decline in the Cool Core fraction in simulated data with redshift up to one.

The understanding of the formation and evolution of cooling flows is fundamental in pursuing cosmological studies with galaxy clusters. It is already known that cool core clusters affect the scaling relations causing a departure from the theoretical expectations and increasing the scatter (Fabian et al. 1994; Zhang et al. 2006). Whether or not we can put together a consistent picture of clusters with and without cool cores will greatly affect the reliability of cluster results in deriving cosmological parameters.
In this paper, we investigate the fraction of cool core clusters in a high-redshift sample imaged with Chandra at $0.7 \leq z<1.4$. To this aim we define and quantify criteria to select clusters as CCC in a sample of nearby clusters, $0.15<z<0.3$, which are then applied to the distant sample. The low- $z$ clusters exhibit markedly different morphologies which is essential to characterize cool cores with varying strength. The low-photon statistics of the distant clusters made a spatially resolved temperature analysis inviable, constraining our study to a spatial analysis of the cluster images. We analyzed the overall surface brightness and determined cooling times in the clusters' central regions. The surface brightness diagnostics were developed based on the nearby sample and were validated to high $-z$ with simulated distant clusters. These were obtained with the cloning technique, a novel method in which we simulate distant galaxy clusters by emulating the appearance of the low- $z$ clusters at the same redshifts of the observed distant sample. Such simulations allow us to make an unbiased comparison of cluster spatial properties between nearby and distant populations.

The two cluster samples are drawn from the Chandra archive. The high- $z$ sample, which is the most relevant for this study, is a statistically-complete sample based on the Rosat Distant Cluster Sample (RDCS), from Rosati et al. (1998). This dataset allows us to draw conclusions on the abundance of $\mathrm{CCC}$ when the Universe was $\sim 1 / 3$ of its age. On the other hand, we constructed the nearby sample by taking morphologically interesting archive clusters, and we used the sample as a test set to understand how we can characterize cool cores.

We note that archive samples will likely be biased in the sense that the majority of the observed clusters are "interesting", meaning showing signs of mergers / disruptions, or on the contrary, having very regular shapes. In addition, cluster selection based on flux limited samples will preferentially find high luminosity/mass clusters at high redshift. This effect, goes in the direction of favoring $\mathrm{CC}$ clusters (which have a bright core) with increasing $z$. For these reasons, constructing complete, unbiased samples to address issues of cluster evolution presents difficulties that should be carefully dealt with. Since the distant and nearby samples are not drawn from a homogenous sample with a known selection function, we do not address the issue of evolution of cool cores on this paper.

As a complement to this X-ray study and with the aim of understanding sample selection effects, we shortly investigate the surface brightness properties of an optically-selected sample of high-z galaxy clusters imaged with Chandra. We assess the incidence rate of cool cores and compare it with the results on the $\mathrm{X}$-ray selected cluster set.

We introduce the cluster samples in Sect. 2, and in Sect. 3 the surface brightness profiles are presented, together with the beta model fitting. In Sect. 4, we introduce the surface brightness concentration parameter $\left(c_{\mathrm{SB}}\right)$ and outline the simulation method we used to test its application. The combined results on the surface brightness profiles and concentration parameter are presented in Sect. 5. The cooling time analysis is described in Sect. 6; Sect. 7 is dedicated to a comparison of X-ray surface brightness properties in optically and X-ray selected high-redshift galaxy clusters. Finally, in Sect. 8 we discuss the results obtained and conclude. The cosmological parameters used throughout the paper are: $H_{0}=70 \mathrm{~km} \mathrm{~s}^{-1} \mathrm{Mpc}^{-1}, \Omega_{\Lambda}=0.7$ and $\Omega_{\mathrm{m}}=0.3$.

\section{Cluster samples}

We used archive Chandra ACIS-I and ACIS-S images in the [0.5-5.0] keV energy range: we selected the 11 low- $z$ clusters 
Table 1. Low redshift cluster sample.

\begin{tabular}{cccccc}
\hline \hline Cluster ID & $z$ & $T(\mathrm{keV})$ & RA (Eq. (J2000)) & Dec (Eq. (J2000) & Exposure (ks) \\
\hline $\mathrm{A}_{1413^{a}}$ & 0.143 & $7.52_{-0.12}^{+0.20}$ & $11: 55: 18.20$ & $+23: 24: 28.80$ & 136 \\
$\mathrm{~A} 907^{a}$ & 0.153 & $5.82 \pm 0.12$ & $09: 58: 22.00$ & $-11: 03: 50.40$ & 100 \\
$\mathrm{~A} 2104^{a}$ & 0.155 & $6.76 \pm 0.19$ & $15: 40: 07.90$ & $-03: 18: 04.00$ & 48 \\
$\mathrm{~A} 2218^{a}$ & 0.177 & $6.25 \pm 0.31$ & $16: 35: 56.00$ & $+66: 12: 45.00$ & 57 \\
$\mathrm{~A} 2163^{b}$ & 0.203 & $12.3_{-1.1}^{+1.3}$ & $16: 15: 45.77$ & $-06: 08: 55.00$ & 80 \\
$\mathrm{~A} 963^{a}$ & 0.206 & $6.02_{-0.19}^{+0.28}$ & $10: 17: 03.40$ & $+39: 02: 51.00$ & 36 \\
$\mathrm{~A} 2261^{a}$ & 0.224 & $7.43_{-0.27}^{+0.49}$ & $17: 22: 27.20$ & $+32: 07: 58.00$ & 32 \\
$\mathrm{~A} 2390^{a}$ & 0.228 & $9.35 \pm 0.15$ & $21: 53: 36.50$ & $+17: 41: 45.00$ & 111 \\
$\mathrm{~A} 1835^{a}$ & 0.253 & $8.09 \pm 0.53$ & $14: 01: 02.00$ & $+02: 52: 40.00$ & 30 \\
$\mathrm{ZwC}^{a} 146^{a}$ & 0.291 & $8.59 \pm 0.39$ & $10: 23: 39.60$ & $+04: 11: 10.00$ & 45 \\
$\mathrm{E} 657^{c}$ & 0.300 & $14.1 \pm 0.2$ & $6: 58: 28.60$ & $-55: 56: 36.03$ & 25 \\
\hline
\end{tabular}

${ }^{a}$ Temperatures taken from Baldi et al. (2007), ${ }^{b}$ temperature from Markevitch et al. (2001), ${ }^{c}$ temperature from Markevitch et al. (2005).

Table 2. High redshift cluster samples.

\begin{tabular}{cccccc}
\hline \hline Cluster ID & $z$ & $T(\mathrm{keV})$ & RA (Eq. (J2000) & Dec (Eq. (J2000)) & Exposure (ks) \\
\hline RX J1221+4918 & 0.700 & $8.4_{-0.8}^{+0.8}$ & $12: 21: 24.50$ & $+49: 18: 14.40$ & 78 \\
RX J1113-2615 & 0.730 & $5.6_{-0.6}^{+0.9}$ & $11: 13: 00.00$ & $-26: 15: 49.00$ & 103 \\
RX J2302+0844 & 0.734 & $8.0_{-1.1}^{+1.2}$ & $23: 02: 48.06$ & $+08: 43: 54.72$ & 108 \\
MS 1137+6625 & 0.782 & $6.8_{-0.5}^{+0.5}$ & $11: 40: 22.81$ & $+66: 08: 14.50$ & 117 \\
RX J1317+2911 & 0.805 & $4.4_{-0.9}^{+1.4}$ & $13: 17: 21.84$ & $+29: 11: 17.00$ & 111 \\
RX J1350+6007 & 0.810 & $4.5_{-0.6}^{+0.7}$ & $13: 50: 47.78$ & $+60: 07: 13.32$ & 58 \\
RX J1716+6708 & 0.813 & $6.9_{-0.7}^{+0.8}$ & $17: 16: 48.76$ & $+67: 08: 25.81$ & 51 \\
MS 1054-0321 & 0.832 & $7.5_{-0.4}^{+0.7}$ & $10: 56: 58.00$ & $-03: 37: 37.30$ & 80 \\
RX J1226+3333 & 0.890 & $12.9_{-1.3}^{+1.4}$ & $12: 26: 58.20$ & $+33: 32: 48.00$ & $9.5+31.5$ \\
CL J1415+3612 & 1.030 & $7.0_{-0.7}^{+0.8}$ & $14: 15: 11.20$ & $+36: 12: 04.00$ & 89 \\
RX J0910+5422 & 1.106 & $6.4_{-1.2}^{+1.5}$ & $09: 10: 45.41$ & $+54: 22: 05.00$ & 170 \\
RX J1252-2927 & 1.235 & $7.3_{-1.0}^{+1.3}$ & $12: 52: 54.50$ & $-29: 27: 18.00$ & 188 \\
RX J0849+4452 & 1.261 & $5.3_{-1.1}^{+1.7}$ & $08: 48: 58.52$ & $+44: 51: 55.08$ & 185 \\
RX J0848+4453 & 1.273 & $2.4_{-1.0}^{+2.5}$ & $08: 48: 36.20$ & $+44: 53: 47.17$ & 185 \\
XMMU J2235-2557 & 1.393 & $6.0_{-1.8}^{+2.5}$ & $22: 35: 20.70$ & $-25: 57: 40.70$ & 189 \\
\hline RCS 1419+5326 & 0.620 & $5.0_{-0.4}^{+0.4}$ & $14: 19: 12.14$ & $+53: 26: 11.56$ & 56 \\
RCS 1107.3-0523 & 0.735 & $4.3_{-0.6}^{+0.5}$ & $11: 07: 24.08$ & $-05: 23: 23.19$ & 93 \\
RCS 1325+2858 & 0.750 & $1.8_{-0.6}^{+1.2}$ & $13: 26: 31.04$ & $+29: 03: 25.02$ & 62 \\
RCS 0224-0002 & 0.778 & $5.1_{-0.8}^{+1.3}$ & $02: 24: 33.61$ & $-00: 02: 24.68$ & 101 \\
RCS 2318.5+0034 & 0.780 & $7.3_{-1.0}^{+1.6}$ & $23: 18: 30.67$ & $+00: 34: 03.03$ & 50 \\
RCS 1620+2929 & 0.870 & $4.6_{-1.1}^{+2.1}$ & $16: 20: 10.01$ & $+29: 29: 15.41$ & 34 \\
RCS 2319.9+0038 & 0.900 & $5.3_{-0.5}^{+0.7}$ & $23: 19: 53.59$ & $+00: 38: 09.05$ & 62 \\
RCS 0439.6-2905 & 0.960 & $1.8_{-0.3}^{+0.4}$ & $04: 39: 37.76$ & $-29: 04: 49.40$ & 92 \\
RCS 1417+5305 & 0.968 & $\ldots$ & $14: 17: 02.13$ & $+53: 05: 23.57$ & 62 \\
RCS 2156.7-0448 & 1.080 & $\ldots$ & $21: 56: 41.63$ & $-04: 47: 53.47$ & 71 \\
RCS 2112.3-6326 & 1.099 & $\ldots$ & $21: 12: 20.51$ & $-63: 26: 13.97$ & 68 \\
\hline
\end{tabular}

${ }^{a}$ The temperature of this cluster was taken from Mullis et al. (2005). Temperatures for RCS clusters refer to Bignamini et al. submitted. All other temperatures refer to the values given by Balestra et al. (2007).

(Table 1) from the sample compiled by Baldi et al. (2007); the high redshift X-ray selected sample, which comprises 15 clusters (first part of Table 2), was drawn from the sample presented in Balestra et al. (2007). We, therefore, refer the reader to these two papers for details in the data processing. In Table 2 we also present the optically-selected distant sample which will be discussed in Sect. 7.

We removed point sources from all images and filled the gaps between the detector chips assuming Poisson statistics, interpolating with neighboring pixels (dividing images by exposure maps is not enough for gap removal because the images are subject to noise propagation). We determined the local background in empty regions of the cluster images; when computing the background a Gaussian filter with a kernel of 1 was applied to the images to avoid zero pixel values. We determined the cluster number counts with the growth curve analysis (Böhringer et al. 2000): cluster counts are calculated in circular apertures until the background level is reached. The maximum value of the growth curve was taken as the nominal cluster number of counts. For each cluster we determined the center-of-mass coordinates, and assigned this value to the cluster center.

\subsection{Low-z sample}

The nearby cluster sample $(0.15<z<0.3$, median $z=0.21)$ is presented in Fig. 1. The selection criteria for its construction were essentially of observational nature, since we were 

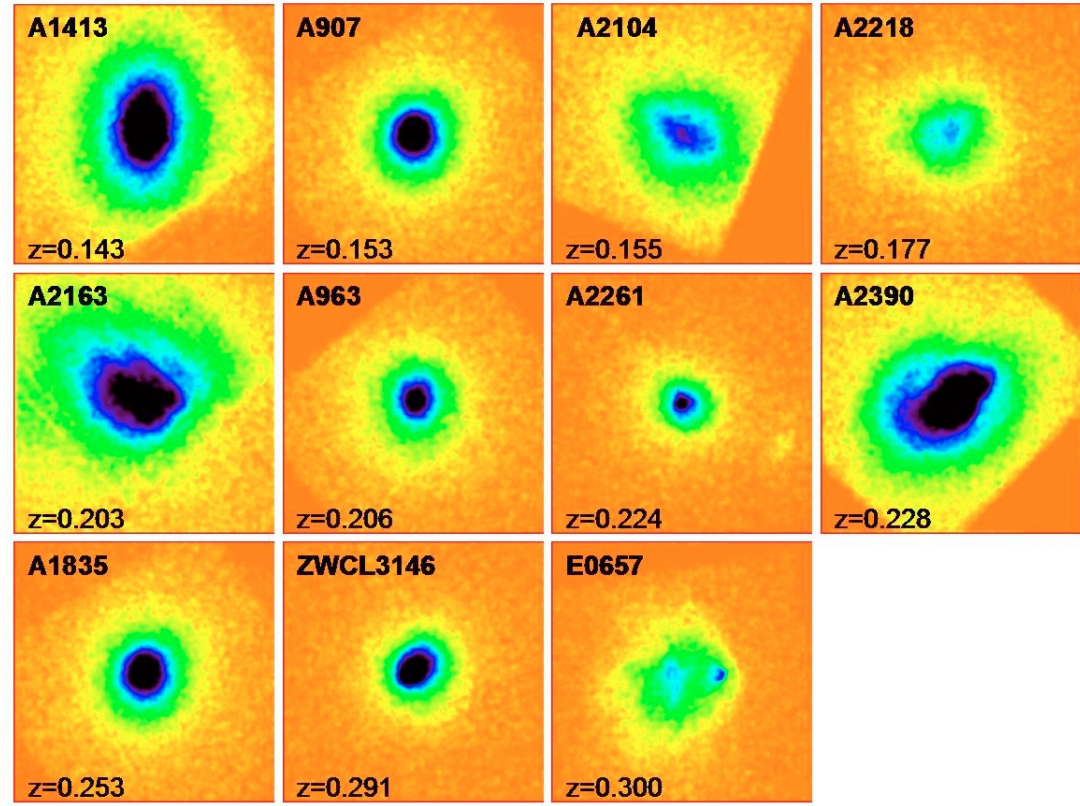



$=0.228$ interested in low-redshift clusters with a size that would properly fit within the field of view of Chandra $\left(16 \operatorname{arcmin}^{2}\right)$, and with sufficiently high number of counts $(>10000)$ to ensure a proper analysis with good enough statistics of the cloned data (see Sect. 4.2 for details on the cloning technique). The sample is heterogeneous in the sense that we wanted to have clusters with known classification in terms of CC/non-CC; we therefore gathered this information from the literature (Vikhlinin et al. 2005; Bauer et al. 2005; Reese et al. 2002; De Filippis et al. 2005; Arabadjis et al. 2002; Pratt \& Arnaud 2002). According to these various sources, clusters A2104, A2218, A2163, and E0657 are obvious non-coolcores whereas A1835 and ZWCL3146 are rare, high luminosity systems, exhibiting pronounced cool cores. Cluster temperatures obtained with spectroscopic fitting were taken mostly from Baldi et al. (2007) and also from Markevitch et al. (2001, 2005) for clusters A2163 and E0657, respectively. Cores were excised when a cool core was present. All systems have $T>5 \mathrm{keV}$.

\subsection{High-z sample}

The distant cluster sample (Fig. 2) is drawn, mostly, from the RDCS (Rosati et al. 1998), with one important addition: a 190 ks Chandra exposure of XMMU J2235.3-2557 (hereafter XMMU J2235), with $z=1.393$. This massive object is the highest redshift cluster in our sample and the second most distant cluster (Mullis et al. 2005) confirmed to date (the most distant cluster known so far has redshift 1.45 (Stanford et al. 2006). The sample ranges from 0.70 to 1.39 in redshift (median $z=0.83$ ), which spans a lookback time between 6.3 and $9.0 \mathrm{Gyr}$, for the assumed cosmology. These are all massive clusters, spanning approximately a decade in mass, with the exception of RX J0848+4453 which has a temperature of $2.4 \mathrm{keV}$. The remaining clusters in the sample have bulk temperatures that range from 4.4 to $12.9 \mathrm{keV}$ and were obtained by spectroscopic fitting. Temperature measurements and general Chandra imaging reduction was extensively described in Balestra et al. (2007). Concerning XMMU J2235, we quote the temperature obtained by Mullis et al. (2005) using XMM-Newton data, although a more precise spectroscopic analysis of the new Chandra data will be available soon (Rosati et al. in preparation). Cluster number counts range from 250 to 11000 with a median value of 1750 counts.

\section{Surface brightness profiles}

\subsection{Scaled surface brightness profiles}

Galaxy clusters are expected to exhibit self-similarity, which allows us to link their physical properties with temperature. This prediction is based solely on gravitational arguments where clusters are described as dark matter dominated entities. However, there has been evidence for a departure from the self-similar scaling scenario (Arnaud et al. 2005b; Chen et al. 2007): the $L_{\mathrm{X}} \propto T^{2}$ scaling relation expected in the case of gravitational only processes, is at odds with the observed $L_{\mathrm{X}} \propto T^{2.88}$ relation. This implies the intervention of nongravitational phenomena that provide extra energy input (Arnaud \& Evrard 1999). An empirical scaling law was then derived which fits to the observations.

For comparison between the local and distant clusters, we scaled the surface brightness profiles according to both the empirical and self-similar scaling relations. In a self-similar, purely gravitational scenario, the surface brightness scales as $S_{\mathrm{X}} \propto$ $T^{0.5}$, whereas in an empirical derivation $S_{\mathrm{X}} \propto T^{1.38}$ (Neumann et al. 1999; Arnaud et al. 2002).

We took into account the surface brightness dimming and considered the redshift evolution of the $L_{X}-T$ and $R-T$ relations (see e.g. Ettori et al. 2004) obtaining:

$S_{\mathrm{X}} h(z)^{-3} \propto T^{\alpha}(1+z)^{4} K_{\mathrm{corr}}$

where $h(z)$, the cosmological factor, is defined as the Hubble constant normalized to its current value, $H_{z} / H_{0}=$ $\sqrt{\Omega_{\mathrm{m}}(1+z)^{3}+\Omega_{\Lambda}}$. The slope $\alpha$ is dependent on the scaling relation and $K_{\text {corr }}$ is the $K$-correction, which is defined in Sect. 4.2.1.

The way in which scaling relations affect our analysis needs some clarification. The purely self-similar gravitational scenario (e.g. Navarro et al. 1996, 1997), which seems to be reasonablywell supported by observations, predicts an evolution of the dark matter halos becoming more compact for fixed enclosed mass 


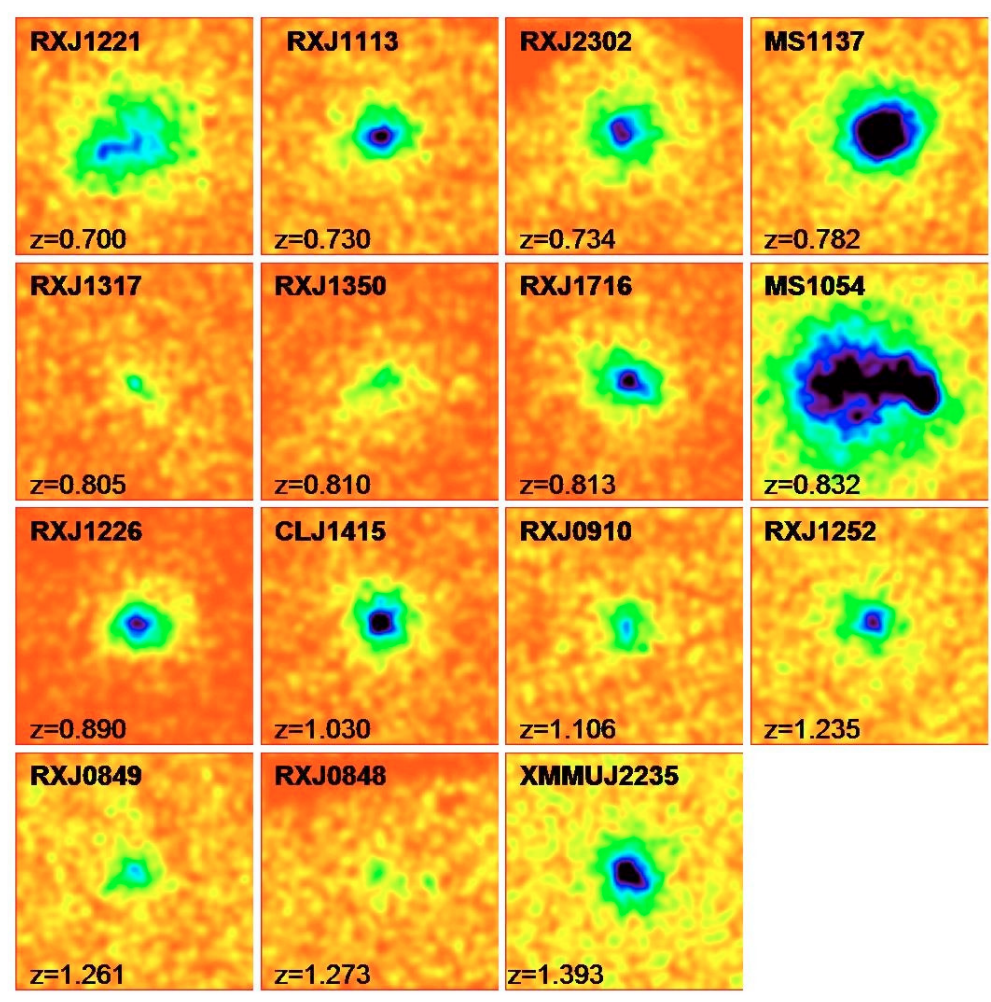

Fig. 2. X-ray selected, distant cluster sample observed with Chandra (point sources are removed). Images are smoothed with a Gaussian of sigma $=3$ and rescaled by the square root of the intensity. Individual boxes have a size of $3 \times 3 \mathrm{arcmin}$. Cluster redshift increases from top-left to bottom-right. with increasing redshift. This is reflected in the global appearance of the ICM and the cluster morphology in X-rays, including further modification due to changes in the ratio of gas to total mass (Arnaud et al. 2002). This intrinsic evolution of the global cluster appearance is accounted for by the above-mentioned empirical scaling relations. Focusing on the study of cool cores in clusters, we are rather more interested in the local physical conditions at the cluster center that are, for example, characterized by the cooling time of the gas or the total radiative energy loss of the ICM in the cool core region. This characterization is not subject to the above-described scaling relations. Thus, if we are interested in comparing the rate at which the central ICM is radiatively cooling in nearby and distant clusters, we have to compare the cluster properties in fixed physical units. We will, therefore, in the following use the empirical scaling relations to reveal changes of the global cluster and cluster ICM structure properties, while we will use unscaled relations to directly compare physical conditions in the cooling cores.

We adopted a fixed density contrast $\Delta=500$ with respect to the critical density of the Universe at the cluster redshift $\left(\rho_{\mathrm{c}}(z)\right)$ to study the global properties of clusters. The fiducial radius, $R_{500}$, was determined using the $R_{500}-T$ relation in Evrard et al. (1996), represented by the following fitting function:

$$
R_{500}=h(z)^{-1} 2.48(k T / 10 \mathrm{keV})^{0.5} H_{50} / H_{70}
$$

Eq. (2) is applicable for clusters with $T>3.5 \mathrm{keV}$.

From the top panels in Fig. 3, it is clear that the low- $z$ profiles converge well for $r>0.2 R_{500}$. However, at high- $z$ two outliers are evident in the self-similar scaling (bottom-right plot): MS 1054-0321 (hereafter MS 1054) and RX J1226+3333. The former is a merging cluster and the latter is the hottest distant cluster. Interestingly, RX J1226+3333 does not appear as an outlier when using the empirical scaling. This reinforces the better use of the empirical relation, which does not boost the clusters at the extreme (highest temperature) outside the bulk of the data points in the relation.

A striking difference between the $\mathrm{CC}$ and non-CC clusters in the nearby sample is evident in Fig. 3 (top panels). The flatness of the profiles of non-cool core clusters A2104, E0657, A2218, and A2163 are in obvious contrast with the remaining profiles that have a much higher central surface brightness - up to nearly 2 orders of magnitude larger - and a steepness that varies according to the strength of the cool core. This result is independent of the choice of scaling. At high redshift this marked distinction is not apparent.

The scatter of the surface brightness profiles scaled with $T^{0.5}$ amounts to $50 \%$ of the average value in the low- $z$ sample at $r=$ $0.3 R_{500}$. In the distant sample, the scatter is boosted by MS 1054 ; if we neglect this outlier we obtain a dispersion around the mean value of $65 \%$ at the same radius.

\subsection{Single and double beta model fitting}

Cluster surface brightness profiles are commonly analyzed with the approximation of the single isothermal beta model (Cavaliere \& Fusco-Femiano 1976). We computed azimuthally-averaged surface brightness profiles in bins of fixed radius, which were fitted with a single $\beta$ model:

$S(r)=S_{0}\left(1+\left(r / r_{\mathrm{c}}\right)^{2}\right)^{-3 \beta+0.5}+C$

where $S_{0}, r_{\mathrm{c}}, \beta$ and $C$ are the central surface brightness, core radius, slope and constant background, respectively. The fitting procedure was performed with a Levenberg-Marquardt leastsquares minimization. 

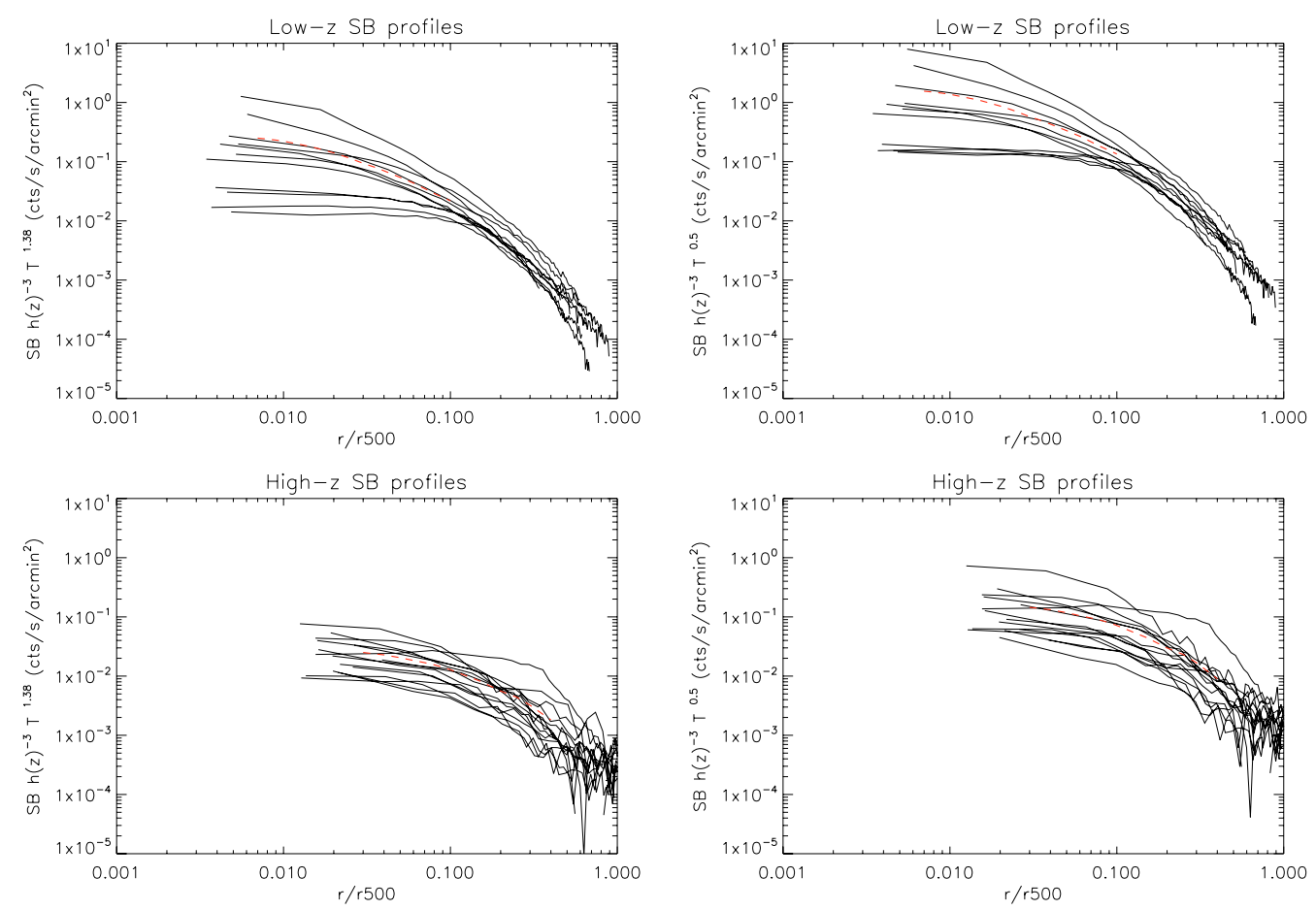

Fig. 3. Left. Empirical scaling of SB profiles - low- $z$ sample (top) and high- $z$ sample (bottom). Right. Self-similar scaling of SB profiles - low- $z$ sample (top) and high- $z$ sample (bottom). The red-dashed lines correspond to the averaged profiles.

When a cluster harbors a cool core, a single beta model is often inappropriate to describe its central excess emission, requiring the use of a $2 \beta$ model:

$S(r)=S_{01}\left(1+\left(\frac{r}{r_{\mathrm{c} 1}}\right)^{2}\right)^{-3 \beta_{1}+0.5}+S_{02}\left(1+\left(\frac{r}{r_{\mathrm{c} 2}}\right)^{2}\right)^{-3 \beta_{2}+0.5}+C$.

Such a conclusion has been reached through observations (Jones \& Forman 1984; Vikhlinin et al. 2005) and is also expected from simulations (Burns et al. 2008). However, this observation cannot be as clearly seen for the high- $z$ clusters because the low statistics provides a good $\chi_{\text {red }}^{2}$ with either a single or a double $\beta$ function. This has been previously noted by Ota $\&$ Mitsuda (2004) who analyzed a sample of 79 clusters with $0.1<z<0.8$, and in Ettori et al. (2004), where nearly all distant clusters in our sample were analyzed. There are however exceptions such as cluster CL J1415+3612 at $z=1.03$, in which the single beta model fails to capture the central SB. A double $\beta$ model is qualitatively more appropriate to describe the core SB excess, although there are no significant differences in the reduced $\chi^{2}$ values of the $1 \beta$ and $2 \beta$ model fit: $\chi_{\text {red }}^{2}=1.043$ in the $1 \beta$ model fit and $\chi_{\text {red }}^{2}=0.954$ in the $2 \beta$ model fit. These results make it obvious that fitting single/double $\beta$ models to quantify the central surface brightness is not conclusive for the high-z sample.

The $\beta$-model parameters will not be used further in our study to characterize CCs, as we found them less useful than the following approaches. We, nevertheless, report our results in Table 3 for comparison with other work. Since the double- $\beta$ model results give, in general, a good representation of the data within the error bars, the model fits also allow for an approximate check and reproduction of our analysis.

\section{Surface brightness concentration}

\subsection{Introducing $c_{\mathrm{SB}}$}

In principle, the most stringent proof for the detection of a cool core is given by the temperature decrease in the core with respect to the bulk of the cluster. Unfortunately, the poor photon statistics does not permit a spatially-resolved spectroscopic analysis of the high- $z$ sample.

Since a central surface brightness excess is a primary indicator (Fabian et al. 1984) of the presence of a cool core, we evaluate the core surface brightness in nearby clusters by defining a concentration parameter as the ratio of the peak over the ambient $\mathrm{SB}$. The aim of this approach is to use a single parameter to make a practical initial classification of cool cores. We chose the apertures corresponding to the peak and the bulk to yield the largest separation between the $\mathrm{CC}$ and non-CC domains. We optimized the $c_{\mathrm{SB}}$ parameter with the low- $z$ sample, varying the radius of the central peak and external radius. The optimal $c_{\mathrm{SB}}$ is found for a peak radius of $40 \mathrm{kpc}$ and a cluster bulk radius of $400 \mathrm{kpc}$ :

$c_{\mathrm{SB}}=\frac{S B(r<40 \mathrm{kpc})}{S B(r<400 \mathrm{kpc})}$.

To support this result, we carried out extensive Monte Carlo simulations of CC and non-CC clusters modelled with, respectively, double and single beta profiles to optimize the choice of the internal and external apertures in the definition of $c_{\mathrm{SB}}$. We explored the range of 30-80 kpc around the typical observed size of cool cores in nearby clusters. We selected an inner radius of $40 \mathrm{kpc}$ to make the separation between $\mathrm{CC}$ and non-CC clusters more obvious. We measured the concentration parameter, as defined in Eq. (5), in simulated clusters and we found that it efficiently separates the two classes of objects.

Defining $c_{\mathrm{SB}}$ in terms of fractions of a scaled radius such as $R_{500}$ proved not to be suitable because the wide range covered in redshift implies sampling cluster regions of various sizes for different redshifts. For example, $0.1 R_{500}$ amounts to $132 \mathrm{kpc}$ for the 
Table 3. Single and double beta model fitting results: core radius, $r_{\mathrm{c}}$, slope $\beta$ and reduced $\chi^{2}$. Errors are not presented when the parameter value is at the limit imposed by the fitting procedure.

\begin{tabular}{|c|c|c|c|c|c|c|c|c|}
\hline \multirow[b]{2}{*}{ cluster } & \multicolumn{3}{|c|}{$\overline{1 \beta \text { fit }}$} & \multicolumn{5}{|c|}{$\overline{2 \beta \text { fit }}$} \\
\hline & $\beta$ & $r_{\mathrm{c}}(\mathrm{kpc})$ & $\chi_{\mathrm{red}}^{2}$ & $\beta_{1}$ & $r_{\mathrm{c} 1}(\mathrm{kpc})$ & $\beta_{2}$ & $r_{\mathrm{c} 2}(\mathrm{kpc})$ & $\chi_{\mathrm{rec}}^{2}$ \\
\hline A1413 & $0.473 \pm 0.002$ & $56 \pm 1$ & 1.821 & $0.755 \pm 0.057$ & $70 \pm 5$ & 0.900 & $286 \pm 2$ & 1.153 \\
\hline A907 & $0.515 \pm 0.002$ & $42 \pm 1$ & 6.360 & $0.635 \pm 0.152$ & $29 \pm 6$ & $0.706 \pm 0.045$ & $131 \pm 16$ & 1.439 \\
\hline A2104 & $0.566 \pm 0.014$ & $161 \pm 6$ & 1.335 & - & - & $0.542 \pm 0.018$ & $162 \pm 7$ & 1.447 \\
\hline A2218 & $0.841 \pm 0.150$ & $222 \pm 30$ & 1.220 & - & - & $0.606 \pm 0.007$ & $159 \pm 4$ & 1.326 \\
\hline A 2163 & $1.003 \pm 0.056$ & $349 \pm 15$ & 1.277 & - & - & $0.517 \pm 0.003$ & $184 \pm 2$ & 2.909 \\
\hline A963 & $0.516 \pm 0.003$ & $68 \pm 1$ & 1.165 & $0.702 \pm 0.063$ & $76 \pm 7$ & 0.900 & $301 \pm 8$ & 1.133 \\
\hline A2261 & $0.540 \pm 0.008$ & $66 \pm 3$ & 1.147 & 1.200 & $49 \pm 11$ & $0.559 \pm 0.006$ & $80 \pm 5$ & 1.090 \\
\hline A 2390 & $0.474 \pm 0.001$ & $46 \pm 1$ & 17.037 & $0.619 \pm 0.020$ & $49 \pm 2$ & 0.900 & $411 \pm 5$ & 2.561 \\
\hline A1835 & $0.528 \pm 0.001$ & $29 \pm 0.3$ & 3.530 & $0.699 \pm 0.050$ & $34 \pm 3$ & $0.719 \pm 0.016$ & $179 \pm 12$ & 1.803 \\
\hline ZwC 13146 & $0.573 \pm 0.001$ & $2 \pm 1$ & 5.012 & $0.639 \pm 0.156$ & $18 \pm 5$ & $0.667 \pm 0.023$ & $93 \pm 9$ & 1.224 \\
\hline E0657 & 1.000 & $477 \pm 4$ & 1.864 & - & - & 0.900 & $433 \pm 3$ & 1.919 \\
\hline RX J1221+4918 & $0.702 \pm 0.067$ & $262 \pm 31$ & 1.285 & - & - & $0.763 \pm 0.062$ & $281 \pm 28$ & 1.321 \\
\hline RX J1113-2615 & $0.668 \pm 0.066$ & $107 \pm 17$ & 1.178 & 1.000 & $98 \pm 46$ & 0.800 & $188 \pm 84$ & 1.048 \\
\hline RX J2302+0844 & $0.451 \pm 0.041$ & $73 \pm 16$ & 1.039 & $0.470 \pm 0.041$ & $68 \pm 8$ & - & - & 1.155 \\
\hline MS $1137+6625$ & $0.807 \pm 0.041$ & $157 \pm 10$ & 1.152 & 1.000 & $77 \pm 25$ & 0.800 & $55 \pm 14$ & 0.961 \\
\hline RX J1317+2911 & $0.537 \pm 0.094$ & $67 \pm 29$ & 1.039 & 1.000 & $52 \pm 38$ & 0.800 & $175 \pm 53$ & 1.020 \\
\hline RX J1350+6007 & $0.561 \pm 0.095$ & $162 \pm 45$ & 1.085 & $0.683 \pm 0.523$ & $36 \pm 27$ & 0.800 & $341 \pm 48$ & 0.909 \\
\hline RX J1716+6708 & $0.740 \pm 0.062$ & $165 \pm 20$ & 0.988 & 1.000 & $28 \pm 6$ & $0.695 \pm 0.071$ & $154 \pm 25$ & 0.930 \\
\hline MS 1054-0321 & 1.000 & $443 \pm 5$ & 2.826 & - & - & 0.800 & $360 \pm 5$ & 2.924 \\
\hline RX J1226+3333 & $0.710 \pm 0.053$ & $131 \pm 16$ & 0.851 & 1.000 & $23 \pm 11$ & $0.727 \pm 0.063$ & $136 \pm 21$ & 0.857 \\
\hline CL J1415+3612 & $0.552 \pm 0.026$ & $61 \pm 8$ & 1.043 & $0.704 \pm 0.094$ & $140 \pm 40$ & 0.800 & $35 \pm 12$ & 0.954 \\
\hline RX J0910+5422 & $0.997 \pm 0.320$ & $177 \pm 57$ & 1.017 & 1.000 & $39 \pm 13$ & 0.800 & $158 \pm 19$ & 1.022 \\
\hline RX J1252-2927 & $0.557 \pm 0.067$ & $89 \pm 21$ & 1.103 & 1.000 & $33 \pm 18$ & $0.601 \pm 0.111$ & $108 \pm 41$ & 1.114 \\
\hline RX J0849+4452 & $0.537 \pm 0.05$ & $72 \pm 19$ & 1.143 & 1.000 & $29 \pm 14$ & 0.800 & $127 \pm 17$ & 1.043 \\
\hline RX J0848+4453 & $0.590 \pm 0.179$ & $122 \pm 69$ & 0.823 & 1.000 & $32 \pm 54$ & 0.800 & $188 \pm 62$ & 1.193 \\
\hline XMMU J2235-2557 & $0.595 \pm 0.028$ & $94 \pm 11$ & 1.197 & 1.000 & $33 \pm 18$ & 0.800 & $155 \pm 11$ & 1.090 \\
\hline
\end{tabular}

most nearby cluster, whereas for the most distant cluster it corresponds to $65 \mathrm{kpc}$. Keeping in mind that the cool core region has been broadly described as the inner $70 \mathrm{kpc}$ of a cluster, it is evident that using fractions of $R_{500}$ will introduce a bias toward lower redshift systems appearing more concentrated. In addition, scaling the radius with temperature would only be meaningful if the cluster samples spanned a different temperature range and/or if there were poor $(T<3.5 \mathrm{keV})$ systems, which is not the case here. Therefore we used a physical radius for measuring the concentration index.

Having thus defined the empirical $c_{\mathrm{SB}}$ parameter with the low- $z$ sample, we investigate whether there is a redshift dependence bias associated with this parameter that would invalidate the comparison of $c_{\mathrm{SB}}$ results between the samples. To this end, we developed a simulation method that clones the observed low- $z$ clusters to high redshift. Measuring the $c_{\mathrm{SB}}$ of simulated distant clusters provides a straightforward way to test for a redshift dependence. We describe this technique in the following section.

\subsection{Testing $c_{\mathrm{SB}}$ : simulating distant galaxy clusters with the cloning method}

The cloning technique has been applied in an optical study by Bouwens et al. (1998), with the purpose of quantifying the evolution of faint galaxies, using low- $z$ galaxies in the Hubble Deep Field. By applying a pixel-by-pixel $K$-correction map to high resolution images of bright galaxies, a set of no-evolution deep fields was created, taking into account the space density and the cosmological volume.

We have revised and adapted the cloning method with the purpose of simulating distant clusters of galaxies as standard nonevolving clusters that can be compared with distant samples. We cloned the low- $z$ sample to the redshifts matching those of the distant sample, and for consistency in the comparison we normalized the simulated cluster counts to the corresponding expected high- $z$ cluster counts, adding the observed high$z$ background as well. Chandra angular resolution is essential for our analysis. Its point spread function (PSF) with a FWHM of $\sim 1$ arcsec corresponds to $8 \mathrm{kpc}$ at $z=1$. Considering that the typical physical size for the $\mathrm{CC}$ region is about an order of magnitude larger than the instrument resolution, there is no need to deconvolve the images with the PSF, which would make the application of our method very difficult. One of the main advantages of this technique is that it is model independent, implying that the simulations are under control, as no tuning of parameters is required. The assessment of the selection effects is rather robust since we only have to rely on the data.

The procedure comprises essentially 2 steps: the flux decrease and the spatial rescaling which the template cluster undergoes.

\subsubsection{Flux dimming: luminosity distance and $K$-correction}

The cluster flux decrease is due to both the physical luminosity distance, $D_{\mathrm{L}}$, and the redshifted energy band. In the adopted cosmology, $D_{\mathrm{L}}$ is given by (see for instance, Carroll et al. 1992; Perlmutter et al. 1997):

$D_{\mathrm{L}}=\frac{c(1+z)}{H_{0}} \int_{0}^{z} h(z)^{-1} \mathrm{~d} z$

The $\mathrm{X}$-ray flux is thus given by

$F_{\mathrm{x}}=\frac{L_{\mathrm{x}}}{4 \pi D_{\mathrm{L}}^{2}}$ 


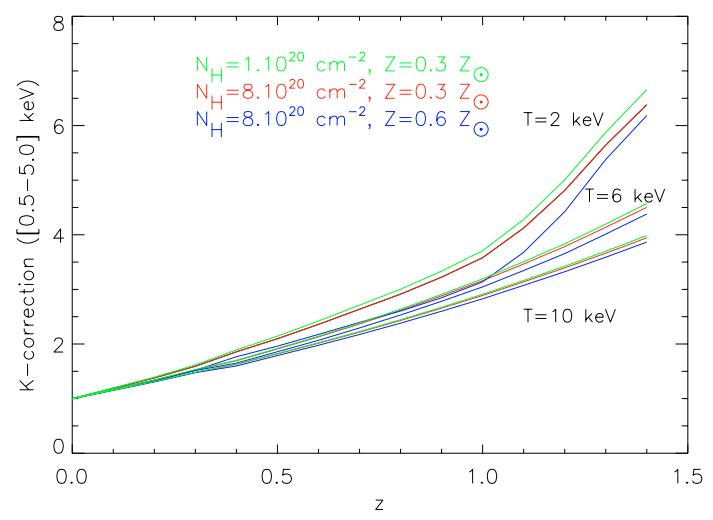

Fig. 4. $K$-correction ratios computed with a MEKAL model: we show the dependence on redshift for temperatures $[2,6,10] \mathrm{keV}$. Red lines refer to an $N_{\mathrm{H}}$ of $8 \times 10^{20} \mathrm{~cm}^{-2}$ and a metallicity of 0.3 solar; blue lines refer to $N_{\mathrm{H}}=8 \times 10^{20} \mathrm{~cm}^{-2}$ and $Z / Z_{\odot}=0.6$; and green lines refer to $N_{\mathrm{H}}=1 \times 10^{20} \mathrm{~cm}^{-2}$ and $Z / Z_{\odot}=0.3$.

where $L_{\mathrm{X}}$ is the X-ray luminosity in the observed frame. Therefore, when cloning to a higher redshift, we must compute the ratio of the squared luminosity distances.

As we are using images in a given energy band $(0.5-5.0 \mathrm{keV})$, a bandpass correction - the $K$-correction - plays an important role in the flux rescale because we are dealing with high redshift values (Hogg et al. 2002). If $f_{v}$ is the unabsorbed spectral flux density, we can express the $K$-correction for an energy band $\left[E_{1}, E_{2}\right]$ as:

$K_{\text {corr }}=\frac{f_{v}\left(z_{1}, \frac{E_{1}}{1+z_{1}}, \frac{E_{2}}{1+z_{2}}\right)}{f_{v}\left(z_{2}, \frac{E_{1}}{1+z_{1}}, \frac{E_{2}}{1+z_{2}}\right)} \frac{\operatorname{ecf}\left(\frac{E_{1}}{1+z_{1}}, \frac{E_{2}}{1+z_{2}}, z_{1}, N_{\mathrm{H}}, Z\right)}{\operatorname{ecf}\left(\frac{E_{1}}{1+z_{1}}, \frac{E_{2}}{1+z_{2}}, z_{2}, N_{\mathrm{H}}, Z\right)}$

where ecf stands for energy conversion factor, a term that relates flux to counts, and depends on the cluster redshift, hydrogen column density, $N_{\mathrm{H}}$, and metallicity, $Z$.

Since the emissivity is not very temperature sensitive in the energy band used for the surface brightness measurements, it is practical and justified to treat the cluster ICM as isothermal. The $K$-correction terms are dependent mostly on the cluster redshift (this dependence is stronger for $z>0.5$ ) and temperature. The latter has a steeper effect for poor systems $(T<3 \mathrm{keV})$ which is not the case here. Applying single cluster temperatures, we computed $K$-corrections with XSPEC v11.3.1 (Arnaud 1996). We used the optically-thin MEKAL model spectra (Kaastra et al. 1992) with photoelectric absorption (WABS(MEKAL)), setting the metallicity to 0.3 solar and $N_{\mathrm{H}}=8 \times 10^{20} \mathrm{~cm}^{-2}$. The spectra were folded with Chandra responses. The metallicity and the $N_{\mathrm{H}}$ have a weak influence in the $K$-correction: at the median redshift and temperature of the high- $z$ sample, using a higher value for the metallicity, e.g., $Z / Z_{\odot}=0.6$, results in a decrease of the $K$-correction by $\approx 8 \%$, and a lower $N_{\mathrm{H}}$ value of $10^{20} \mathrm{~cm}^{-2}$ has the marginal effect of raising the $K$-correction by $\approx 2 \%$ - see Fig. 4 for a description of how the $K$-correction varies in these parameters.

Equation (8) thus becomes:

$K_{[0.5-5.0] \mathrm{keV}}=\frac{\int_{0.5}^{5.0} f_{v} \mathrm{~d} v}{\int_{0.5(1+z)}^{5.0(1+z)} f_{v} \mathrm{~d} v}$

so that $L_{\mathrm{X}}=L_{\mathrm{X} 0} / K_{[0.5-5.0]}$, where $L_{\mathrm{X} 0}$ is the rest frame luminosity of the source. The calculated correction ratios range from a minimum of 1.5 , when we clone a high temperature cluster at

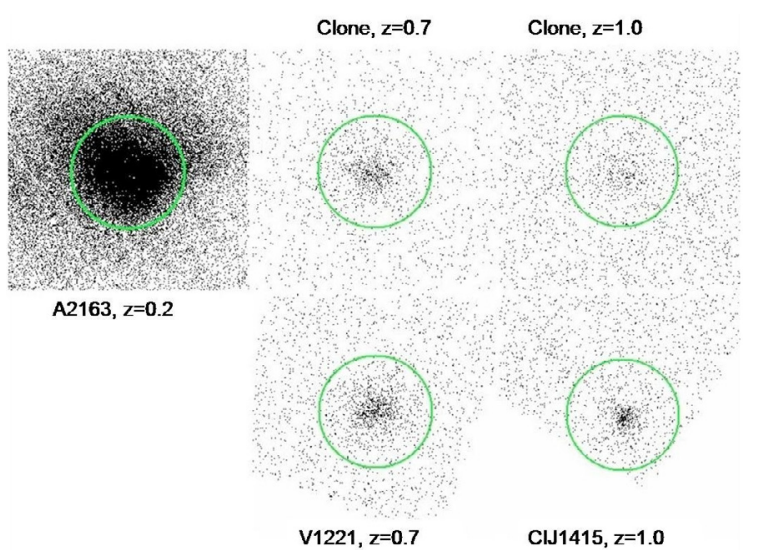

Fig. 5. Cloning example: Chandra image of low-redshift non-CC cluster, A2163, and 2 cloned images (top), compared with a non-cool core cluster at $z=0.7, \mathrm{RX} \mathrm{J1221} \mathrm{(bottom} \mathrm{left),} \mathrm{and} \mathrm{a} \mathrm{CC} \mathrm{cluster} \mathrm{at} z=1.0$, CL J1415 (bottom right). Green circles have a radius of 2 arcmin.

$z \sim 0.3$ to a final redshift of 0.7 , and may reach a maximum of 4 when the cloning redshift is the largest value, i.e, 1.393. If a second temperature component related to the cluster core were considered, this would increase the $K$-correction by a factor of $10-20 \%$.

\subsubsection{Spatial rescaling}

The spatial rescale that a cluster undergoes when cloned to a higher redshift corresponds to the ratio of the angular distances, $D_{A}=D_{\mathrm{L}} /(1+z)^{2}$. Since the premise of this method is nonevolution of the clusters, it should be clear that the size rescale does not account for the shrinking expected when applying the cosmological factor $h(z)$, which comes about as an intrinsic evolution of clusters. The spatial resizing was implemented with a gridding linear interpolation. Note that, as explained above, apart from the redshift resizing, no additional size scaling of the kind of Eq. (2) was applied, as we compare the cluster cores in physical units.

To illustrate this method, we show, in Fig. 5, an example of cloning. We clone A2163, a non-cool core cluster at $z=0.2$, to redshifts 0.7 and 1.0. The observed high- $z$ clusters at the same redshifts (RX J1221 at $z=0.7$ and CL J1415 at $z=1.0$ ) are displayed in the lower panels. The simulated X-ray images were normalized to have an identical number of counts as the corresponding high- $z$ distant clusters, and the same background, which were measured as described in Sect. 2.

A simple consistency check, which allows us to evaluate the procedure resides in measuring the surface brightness within a physical aperture, both in the real and simulated image. For a given energy band we should obtain a ratio of the integrated flux which scales as follows:

$\frac{S_{\mathrm{X}}\left(z_{i}\right)}{S_{\mathrm{X}}\left(z_{f}\right)}=\frac{\left(1+z_{f}\right)^{4}}{\left(1+z_{i}\right)^{4}} K_{\text {corr }}$.

We recover perfect agreement of the results from the two approaches (within the expected numerical errors).

\subsection{The $\mathrm{C}_{\mathrm{SB}}$ distribution}

We find a very good agreement between the surface brightness concentration of the clones and the parent (low- $z$ ) $c_{\mathrm{SB}}$ distribution, implying no redshift dependence in $c_{\mathrm{SB}}$. This can be exemplified by making 10 realizations of cloning the complete 


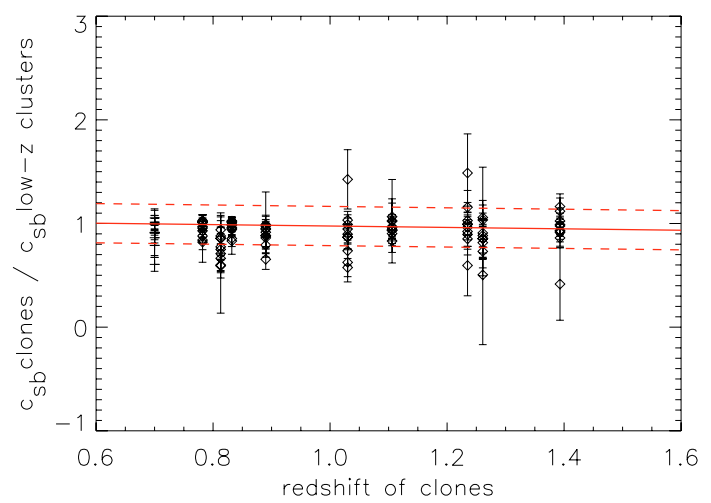

Fig. 6. Analysis of 10 realizations of cloning the nearby cluster sample to high- $z$ : we plot the ratio $c_{\mathrm{SB}}$ [clones] $/ c_{\mathrm{SB}}$ [lowz] as a function of cloning redshift. Individual error bars refer to 1-sigma confidence level. The red-solid line represents the linear fit to the data and corresponding 1 -sigma errors are shown in dashed line.

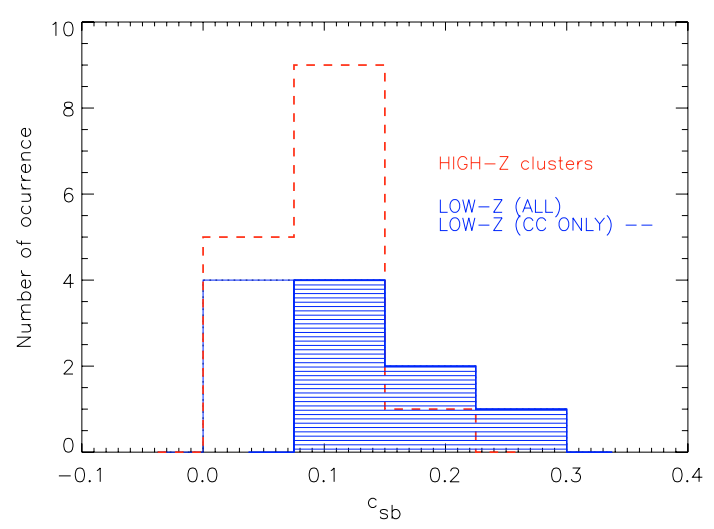

Fig. 7. Histograms of $c_{\mathrm{SB}}$ for the low- $z$ sample (blue solid line with the known CC clusters indicated in hatched) and for the high- $z$ sample (red dash line).

nearby sample to $0.7 \leq z<1.4$ and computing the ratio $c_{\mathrm{SB}}[$ clones $] / c_{\mathrm{SB}}[$ low $-z]$ as a function of redshift. This is illustrated in Fig. 6: the ratio of $c_{\mathrm{SB}}$ randomly fluctuates around one with no trends in redshift, which is confirmed by the weighted mean linear fit to the data. Moreover, no dependence with temperature was found. This indicates that $c_{\mathrm{SB}}$ is an unbiased quantity which can be applied to the real high- $z$ sample, therefore we can safely compare the nearby with the distant samples using $c_{\mathrm{SB}}$.

The distribution of $c_{\mathrm{SB}}$ for both the nearby and distant cluster samples is presented in Fig. 7: the histogram of the low$z$ sample is shown in blue with the CCC in blue-hatched and the high- $z$ sample is represented in red. A Kolmogorov-Smirnov (KS) test yields a probability of $45 \%$ that both $c_{\mathrm{SB}}$ distributions were drawn from the same population, implying there is no significant difference between the two samples with respect to the $c_{\mathrm{SB}}$ parameter. We measured $c_{\mathrm{SB}}$ by performing aperture photometry in central regions enclosing 40 and $400 \mathrm{kpc}$. Errors were determined using Poisson statistics - see Table 4 for a listing of these values.

\section{Stacked SB profiles in bins of $c_{\mathrm{SB}}$}

Owing to the low number of counts of the distant cluster sample, we resorted to stacking the scaled SB profiles that we computed in Sect. 3.1, making use of the $c_{\mathrm{SB}}$ results (Table 4).
Table 4. Surface brightness concentration, $c_{\mathrm{SB}}$ of the nearby and distant samples.

\begin{tabular}{cccc}
\hline \hline Low- $z$ ID & $c_{\mathrm{SB}}$ & High- $z$ ID & $c_{\mathrm{SB}}$ \\
\hline $\mathrm{A} 1413$ & $0.095 \pm 0.001$ & RX J1221+4918 & $0.028 \pm 0.004$ \\
$\mathrm{~A} 907$ & $0.169 \pm 0.002$ & RX J1113-2615 & $0.095 \pm 0.012$ \\
$\mathrm{~A} 2104$ & $0.044 \pm 0.001$ & RX J2302 +0844 & $0.072 \pm 0.009$ \\
$\mathrm{~A} 2218$ & $0.042 \pm 0.001$ & MS 1137+6625 & $0.096 \pm 0.007$ \\
$\mathrm{~A} 2163$ & $0.024 \pm 0.002$ & RX J1317+2911 & $0.123 \pm 0.030$ \\
$\mathrm{~A} 963$ & $0.101 \pm 0.002$ & RX J1350+6007 & $0.057 \pm 0.012$ \\
$\mathrm{~A} 2261$ & $0.111 \pm 0.003$ & RX J1716+6708 & $0.082 \pm 0.010$ \\
$\mathrm{~A} 2390$ & $0.120 \pm 0.001$ & MS 1054-0321 & $0.016 \pm 0.002$ \\
$\mathrm{~A} 1835$ & $0.236 \pm 0.003$ & RX J1226+3333 & $0.083 \pm 0.011$ \\
ZW 13146 & $0.217 \pm 0.003$ & CL J1415+3612 & $0.151 \pm 0.015$ \\
$\mathrm{E} 0657$ & $0.017 \pm 0.001$ & RX J0910+5422 & $0.101 \pm 0.021$ \\
& & RX J1252-2927 & $0.088 \pm 0.014$ \\
& & RX J0849+4452 & $0.099 \pm 0.023$ \\
& & RX J0848+4453 & $0.064 \pm 0.030$ \\
& & XMMU J2235-2557 & $0.103 \pm 0.012$ \\
\hline
\end{tabular}

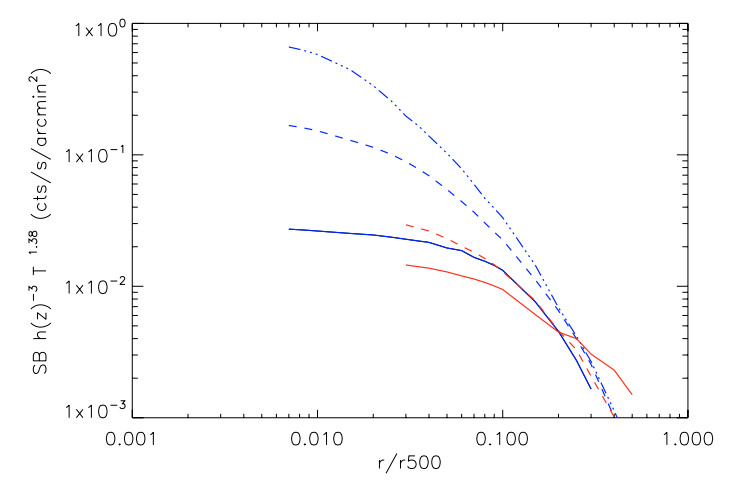

Fig. 8. Stacked surface brightness profiles according to $c_{\mathrm{SB}}$ bins: the nearby sample (blue) presents 3 bins: non-cool core (solid, bin 1), moderate CC (dash, bin 2), and strong CC (dash-dot, bin 3); the distant sample (red) shows 2 bins: non-cool core (solid, bin 1) and moderate CC (dash, bin 2).

From Fig. 7, we define three categories of cool cores: non$\mathrm{CC}\left(c_{\mathrm{SB}}<0.075\right)$, moderate $\left(0.075<c_{\mathrm{SB}}<0.155\right)$, and pronounced $\left(c_{\mathrm{SB}}>0.155\right) \mathrm{CC}$.

Figure 8 shows the stacked profiles of both samples scaled according to the empirical scaling law, in units of $R_{500}$. At high $-z$ we are limited by the spatial resolution of Chandra up to $0.03 R_{500}$, and at low- $z$ we are constrained by the background and cluster size to probe regions up to $0.5 R_{500}$ (with the exception of A2104, which can be traced only as far as $0.3 R_{500}$ ). We excluded the clusters with complex morphology due to mergers (E0657 at low- $z$ and MS 1054 at high- $z$ ) from the stacking procedure because they introduce strong deviations from the average profile. These deviations which are related to the difficulty of identifying the cluster center. The stacked profiles exhibit different shapes (slope and central emission) consistent with Fig. 7, where the low- $z$ sample spans over 3 bins of $c_{\mathrm{SB}}$ whereas the high- $z$ sample covers the first 2 . Both the low- and high-redshift profiles are in full agreement on the outskirts at $r \approx 0.3 R_{500}$ (the deviation of the high- $z$ NCC profile (bin 1 ) at large radius is mostly due to the noise of the individual profiles), but not at the centers where we find a central emission offset: at $r / R_{500}=0.03$ the SB of bin 1 is $0.023 \pm 0.005$ and $0.014 \pm 0.006 \mathrm{cts} / \mathrm{s} / \mathrm{arcmin}^{2}$ for the low- $z$ and high- $z$ samples, respectively, with a ratio $\mathrm{SB}($ low $-z) / \mathrm{SB}($ high $-z)=1.6$. At the same radius the offset is larger for the second bin: $\mathrm{SB}=0.089 \pm 0.026 \mathrm{cts} / \mathrm{s} / \mathrm{arcmin}^{2}$ for nearby clusters and $0.029 \pm 0.019 \mathrm{cts} / \mathrm{s} / \mathrm{arcmin}^{2}$ for distant 


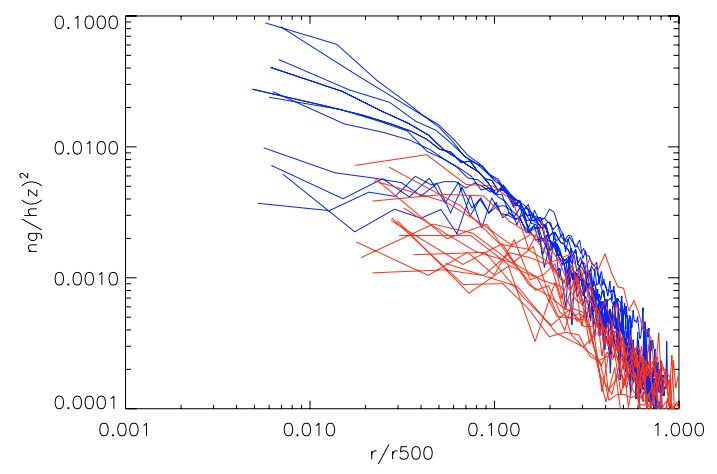

Fig. 9. Scaled density profiles: low- $z$ (blue) and high- $z$ (red) clusters.

clusters, with a corresponding ratio of 3.0. The quoted errors refer to the standard deviations associated with the stacking process, which are larger than the combined measurement errors.

The central surface brightness clearly does not follow the expected predictions from scaling relations. As mentioned above, it may be more meaningful to compare the physical state of the ICM directly, thus dropping the temperature and redshift $(h(z))$ scaling. In this case, we obtain a closer match between the low- $z$ and high- $z$ profiles, indicating that the physical conditions are similar.

\section{Cooling time analysis}

The radiative cooling of the intracluster gas originates mainly from thermal bremsstrahlung emission, with additional line and recombination radiation. The cooling time is defined as $\mathrm{d} t / \mathrm{d} \ln T_{\text {gas }}$ (Sarazin 1988) and allows the evaluation of the cooling rate in galaxy clusters. Adopting a gas enthalpy model for the cooling function, $t_{\text {cool }}$ can be approximated by:

$t_{\text {cool }}=\frac{2.5 n_{\mathrm{g}} T}{n_{\mathrm{e}}^{2} \Lambda(T)}$

where $\Lambda(T), n_{\mathrm{g}}, n_{\mathrm{e}}$ and $T$ are the cooling function, gas number density, electron number density and temperature respectively (Peterson \& Fabian 2005), and with $n_{\mathrm{g}}=1.9 n_{\mathrm{e}}$. The central cooling time is a sensitive parameter to characterize a cool core: when a cool core forms, the central temperature decreases and conversely the central density increases, resulting in a small cooling time. Thus, we expect that CCC present cooling times shorter than the Hubble time or the time since the last major merger event.

\subsection{Density profiles}

Gas density profiles were obtained by deprojection of the surface brightness profiles. We corrected the gas density for the cosmological expansion: attending the $M_{\text {gas }}-T$ and $R-T$ scaling relations (Arnaud et al. 2005a) it follows that $n_{\text {gas }}$ should be scaled by $h(z)^{-2}$ to account for the more compact shape of distant clusters. The density profiles of both cluster populations are presented in Fig. 9. Similar to what we found earlier in the scaled surface brightness profiles, the high- $z$ density in the core region is also systematically lower than the central low- $z$ gas density. This should be due to the $h(z)$ scaling, as explained earlier in Sect. 5.

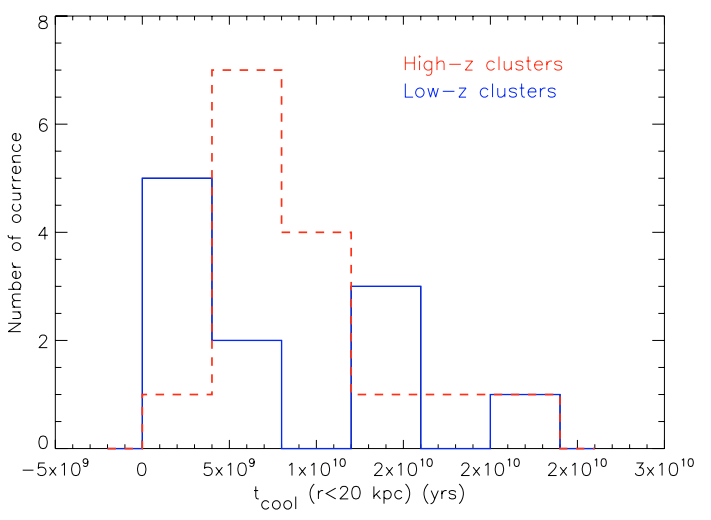

Fig. 10. Distribution of the cooling time, $t_{\text {cool }}$, in the low- $z$ (blue) and high- $z$ (red) samples.

Table 5. Cooling time, $t_{\text {cool }}$ measured at $20 \mathrm{kpc}$, of the nearby and distant samples.

\begin{tabular}{cccc}
\hline \hline Low- $z$ ID & $t_{\text {cool }}(\mathrm{Gyr})$ & High- $z$ ID & $t_{\text {cool }}(\mathrm{Gyr})$ \\
\hline A1413 & $5.50_{-0.17}^{+0.23}$ & RX J1221+4918 & $20.60_{-4.61}^{+4.55}$ \\
A907 & $2.91 \pm 0.10$ & RX J1113-2615 & $7.01_{-1.38}^{+1.75}$ \\
A2104 & $15.10 \pm 0.99$ & RX J2302+0844 & $14.70_{-3.67}^{+3.85}$ \\
A2218 & $12.10 \pm 1.22$ & MS 1137+6625 & $6.38_{-0.83}^{+0.86}$ \\
A2163 & $15.10_{-1.81}^{+2.06}$ & RX J1317+2911 & $8.77_{-3.36}^{+4.17}$ \\
A963 & $4.52_{-0.26}^{+0.32}$ & RX J1350+6007 & $10.90_{-3.24}^{+3.65}$ \\
A2261 & $3.89_{-0.26}^{+0.38}$ & RX J1716+6708 & $6.39_{-1.25}^{+1.34}$ \\
A2390 & $2.67 \pm 0.07$ & MS 1054-0321 & $16.70_{-2.43}^{+3.25}$ \\
A1835 & $1.13 \pm 0.09$ & RX J1226+3333 & $4.11_{-0.83}^{+0.88}$ \\
ZwC13146 & $1.32 \pm 0.08$ & CL J1415+3612 & $3.90_{-0.71}^{+0.72}$ \\
E0657 & $20.80 \pm 1.62$ & RX J0910+5422 & $8.65_{-2.71}^{+3.11}$ \\
& & RX J1252-2927 & $7.35_{-1.78}^{+2.01}$ \\
& & RX J0849+4452 & $9.02_{-3.11}^{+4.12}$ \\
& & RX J0848+4453 & $6.19_{-4.00}^{+7.87}$ \\
& & XMMU J2235-2557 & $4.84_{-1.82}^{+2.38}$ \\
\hline
\end{tabular}

\subsection{Cooling time at $r=20 \mathrm{kpc}$}

The cooling time was measured within a central aperture of $20 \mathrm{kpc}$ radius extrapolating the density profiles with a $1 \beta$ model. We found it preferable to use an aperture with a fixed physical radius for a consistent analysis of all clusters, as we are limited by the instrument's resolution at high redshift. Using a fraction of a scaled radius was not suitable as the redshift range is fairly large, and therefore we would probe larger areas at low redshift, introducing a bias. As a result, the strong cool cores would have a (artificially) boosted cooling time by a factor $\sim 2-3$.

Since temperature profiles cannot be derived from high- $z$ data, we used the spectral fit temperature in the single temperature (MEKAL) model for the cooling time computation. As a result, the $t_{\text {cool }}$ values in this case are an upper limit because in the presence of cool cores the central temperature would be lower, decreasing the cooling time.

The cooling time distribution is plotted in Fig. 10 and presented in Table 5; we obtained errors from the propagation of the errors on the temperature and $n g$. Four low- $z$ clusters (A1835, ZwC13146, A2390 and A907) present a cooling time <3 Gyr and the 4 nearby non-CC show a $t_{\text {cool }}>10$ Gyr. At high- $z$ there is no system with $t_{\text {cool }}<3 \mathrm{Gyr}$, although the majority $(11 / 15)$ has $t_{\text {cool }}<10 \mathrm{Gyr}$, with only 4 clusters presenting a $t_{\text {cool }}>10 \mathrm{Gyr}$. 


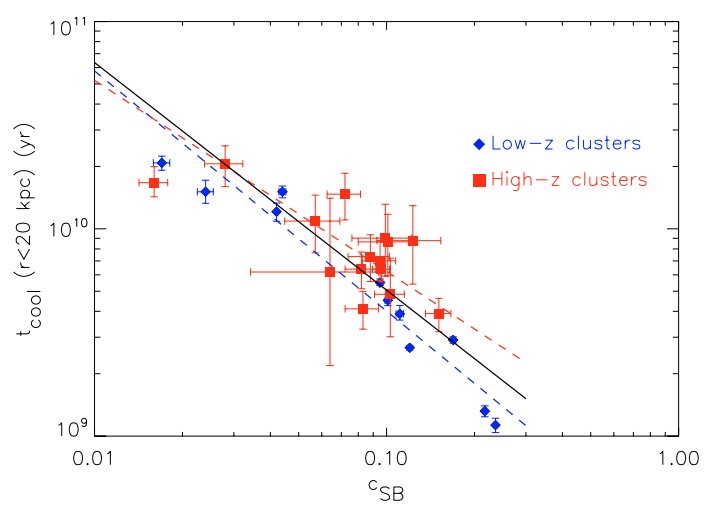

Fig. 11. Correlation between cooling time, $t_{\text {cool }}$ and the surface brightness concentration, $c_{\mathrm{SB}}$, for the low- $z$ (blue diamonds) and high- $z$ (red squares) samples. The black-solid line refers to the composite (both populations) bootstrap fit; the blue- and red-dashed lines refer to separate fits to the nearby and distant samples, respectively.

While similar cooling times or cooling rates in the nearby and distant samples point to similar physical conditions in the central cluster regions, the different ages of the clusters lead to some different conclusions of these results. The cooling rates for more than half of the nearby clusters are shorter than their estimated ages ( $\sim 10 \mathrm{Gyr})$ which implies that they approach a steady state of cooling, some mass deposition, mass inflow and reheating by central AGN. With the same cooling time distribution, only a small fraction of the distant clusters (with ages between 5-8 Gyr) will have a chance to reach this steady state. If we therefore use the classical definition of cooling flows in which $t_{\text {cool }}<t_{\text {age }}$ also for the distant clusters, we find that the fraction of CCs is $4 / 15$, which is significantly smaller than the ratio found for nearby clusters (e.g., Peres et al. 1998; Bauer et al. 2005; Chen et al. 2007).

\subsection{The correlation $t_{\mathrm{cool}}-c_{\mathrm{SB}}$}

As we are interested in evaluating the degree of cooling of the central intracluster gas with a simple function that can be deduced from imaging data only, we investigated the relation between the cooling time and the surface brightness concentration. We found a strong negative correlation with a Spearman's $\rho$ rank correlation coefficient of -0.84 and a significance of non-correlation $p=8.15 \times 10^{-8}$. Using 1000 bootstrap samples, we performed a linear fit in the log-log plane of $t_{\text {cool }}$ and $c_{\mathrm{SB}}$, from which we derived an average slope of the powerlaw function that describes the correlation between the two cooling estimators. A composite fit to both cluster samples yields $t_{\text {cool }} \propto c_{\mathrm{SB}}^{-1.10 \pm 0.15}$, (Fig. 11). Fitting the nearby and distant samples separately yields the following correlations: $t_{\text {cool }}\left(\right.$ high $\left._{z}\right) \propto$ $c_{\mathrm{SB}}^{-0.92 \pm 0.26}$ and $t_{\text {cool }}\left(\operatorname{low}_{z}\right) \propto c_{\mathrm{SB}}^{-1.16 \pm 0.14}$.

${ }^{\mathrm{B}}$ Using the single fit correlation we obtain the cooling time at the reference thresholds $c_{\mathrm{SB}}=0.075$ and $c_{\mathrm{SB}}=0.155$, as $6.9 \mathrm{Gyr}$ and 3.1 Gyr, respectively.

\section{X-ray versus optically selected distant clusters: a comparison using $c_{\mathrm{SB}}$}

In this section, we briefly analyze the X-ray surface brightness properties of distant galaxy clusters detected in the optical. Specifically, we evaluate the degree of cooling using the previously defined $c_{\mathrm{SB}}$ parameter and compare this degree with the results obtained for the X-ray selected high redshift sample.

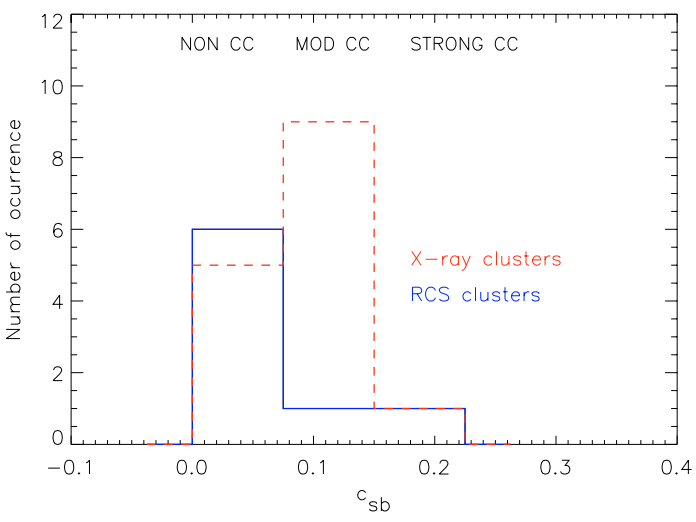

Fig. 12. Histogram of $c_{\mathrm{SB}}$ for the RCS sample in solid blue line; the high- $z$ X-ray sample is overplotted in red dashed line.

The Red Sequence Cluster Survey (RCS) aims at finding and characterizing distant galaxy clusters using $R$ and $z$-band imaging, up to redshift $z=1.4$ (Gladders \& Yee 2005). Selecting clusters in the optical allows us to study high redshift poor systems with low temperature, which are usually not found in flux limited samples restricted to high $L_{\mathrm{X}}$ objects at high redshift.

Although there are indications of a large scatter in the $L_{X}-T$ relation of the RCS sample, such clusters with low temperature (median $T=5 \mathrm{keV}$ ) enable us to probe a larger dynamical range in the $L_{\mathrm{X}}-T$ relation.

The connection between optical and X-ray characteristics of these distant clusters has been under investigation (Hicks et al. 2007). Currently, 11 clusters with $0.6<z<1.2$ have been observed with Chandra and X-ray properties as temperature and luminosity have been derived (Hicks et al. 2007; Bignamini et al., submitted). We based our analysis on Chandra images reduced by Bignamini et al. - see Table 2 for an overview of the sample.

We find that 3 of the 11 RCS clusters are barely detected in $\mathrm{X}$-rays and have such a low surface brightness that a $c_{\mathrm{SB}}$ analysis is not possible. The measurement of the surface brightness concentration as described in Sect. 4.3, yields that the majority (6 of 8) of the RCS clusters with measurable X-ray emission have $c_{\mathrm{SB}}<0.075$, thus falling in the non-CC regime. Of the remaining two: $\mathrm{RCS} 1107$ has a $c_{\mathrm{SB}}=0.143$ indicating a moderate $\mathrm{CC}$, and $\mathrm{RCS} 1419$ has $c_{\mathrm{SB}}=0.185$, suggesting a strong $\mathrm{CC}$ (see histogram in Fig. 12). The $c_{\mathrm{SB}}$ distribution of the X-ray distant clusters is overplotted in the same figure (red line) for direct comparison. The Kolmogorov-Smirnov statistic between the two $c_{\mathrm{SB}}$ distributions is 0.4 , with a probability of the samples being drawn from the same distribution of $20 \%$. With such low statistics, the KS test does not provide a sensitive distinction on these two datasets.

The outcome of this analysis, based on our small samples suggests that high- $z$ optically selected clusters may have a lower fraction of cool cores with respect to clusters detected in X-rays. Larger samples are needed to draw conclusions on possible different physical conditions of the ICM in optically-selected clusters, which may have not reached a final state of virialization

\section{Discussion and conclusions}

In this paper we investigate the detection and quantify the strength of cool cores in the high redshift cluster population. We obtained azimuthally-averaged SB profiles and scaled them, in this manner accounting for different cluster temperatures. The 
segregation of cool cores at low redshift using this method is evident in Fig. 3 (top panels). However, this segregation is not obvious in the distant sample, and the use of single/double $\beta$ model fitting does not provide conclusive results.

A surface brightness concentration index, $c_{\mathrm{SB}}$, was then defined and measured in the local sample, which efficiently separates the non-cool core from the cool core regime. This is in agreement with previously published results on the cuspiness of these clusters. In Hashimoto et al. (2007), a similar surface brightness concentration was defined, using the ratio of elliptical aperture radii enclosing, respectively, 30 and $100 \%$ of the cluster surface brightness. This parameter was applied to a sample of 101 clusters with $0.05<z<1$, these including 4 of the 11 nearby clusters and 12 of the 15 distant clusters we study here. Comparing the results from both measurements for individual clusters we find a good agreement with our results, with the exception of A1835.

We also developed a robust, parameter-free method to simulate galaxy clusters at redshift $z \geq 0.7$, making use of the heterogeneous nearby $(0.15<z<0.3)$ sample. These simulated clusters serve as a benchmark for nonevolving clusters, which allow us to test the suitability of indicators of cool cores at high redshift. Using our cloning technique, we verified the redshift independence of $c_{\mathrm{SB}}$, thus making it a simple, unbiased quantity to study cool cores in the distant cluster sample (Fig. 7). The analysis of the concentration and radial profiles allows us to define three $c_{\mathrm{SB}}$ bins, which distinguish different regimes: non$\mathrm{CC}\left(c_{\mathrm{SB}}<0.75\right)$, moderate $\mathrm{CC}\left(0.75<c_{\mathrm{SB}}<1.55\right)$ and strong $\mathrm{CC}\left(c_{\mathrm{SB}}>1.55\right)$. By stacking the profiles in these bins (Fig. 8), we obtained a robust classification of cool cores, indicating the range of different cooling rates the two cluster samples span. This is particularly useful for the distant sample that is affected by low X-ray count statistics.

From the overall surface brightness analysis we conclude that the majority (10/15) of the high- $z$ clusters present mild cool cores, similar to those found in the nearby sample. In addition to the surface brightness analysis, we measured the clusters' cooling time in a central region with $20 \mathrm{kpc}$ radius, using a single global temperature. Analyzing the reference, low- $z$ sample, we conclude that strong cool cores present $t_{\text {cool }}<3 \mathrm{Gyr}$, whereas moderate CC have $3<t_{\text {cool }}<10$ Gyr and finally non-CC show $t_{\text {cool }}>10$ Gyr. The majority $(11 / 15)$ of the high- $z$ clusters are characterized by $3<t_{\text {cool }}<10$ Gyr with only 4 systems showing $t_{\text {cool }}>10$ Gyr. We found no distant clusters with $t_{\text {cool }}<3$ Gyr. Similar cooling time bins were provided by Bauer et al. (2005), to discriminate between strong, mild and non-cool core systems. These authors measured the central cooling time and also the cooling time at $50 \mathrm{kpc}$ : good agreement on their $t_{\text {cool }}$ at $50 \mathrm{kpc}$ and our $t_{\text {cool }}$ at $20 \mathrm{kpc}$ is found for the common objects in the intermediate- $z$ sample of Bauer et al. (2005) (4/6 objects within $1 \sigma$ errors). Although the distribution of the cooling time is quite similar in the nearby and distant samples, a smaller fraction of distant clusters has $t_{\text {cool }}<t_{\text {age }} \sim t_{\text {Hubble }}$, and therefore the fraction of distant CCs following the classical cooling flow distribution is smaller than for local clusters.

We find a strong correlation between $t_{\mathrm{cool}}$ and $c_{\mathrm{SB}}$, which allow us to reliably relate a physical quantity with a phenomenological parameter. This correlation is described by a power-law fit: $t_{\text {cool }} \propto c_{\mathrm{SB}}^{-1.10 \pm 0.15}$ (Fig. 11). The correlation does not change significantly if we fit the two samples separately. The low- $z$ clusters show a slightly steeper slope $(-1.16 \pm 0.14)$ when compared with the high $-z$ slope $(-0.92 \pm 0.26)$.

Investigating galaxy cluster properties at high redshift is difficult as these systems have small angular size, requiring high resolution instruments and long integration time, particularly for spectroscopy. It is, therefore, understandable that few attempts have been made to probe the centers of distant galaxy clusters. Vikhlinin et al. (2006) presented a study on the evolution of $\mathrm{CCC}$ in the redshift range [0.5-0.9], which they compared to a local sample. They found evidence for evolution of the $\mathrm{CC}$ fraction, with a lack of cool cores in the distant sample. They use a different indicator for the surface brightness cuspiness, which might have a lower sensitivity to moderate cool core systems. A meaningful comparison with our study would require a detailed analysis of the different cool core estimators.

One often finds that massive, luminous galaxies containing AGN, lie at the centers of cool core clusters (Eilek 2003). In an attempt to establish such a correlation we searched for cD galaxies in the cluster samples, examining available optical images. We find that, in the low- $z$ sample, all CCC indeed host a bright, massive galaxy, but also A2104, a non-cool core cluster, possesses a cD galaxy with an associated AGN (Liang et al. 2000; Martini et al. 2004). Could this be a sign of an AGN heating overshoot that could have contributed to destroy the cool core? At high redshift there is no obvious trend: the majority of the clusters possess a cD galaxy but it does not correlate with $c_{\mathrm{SB}}$.

In the established scenario of hierarchical structure formation, galaxy clusters develop through mergers and by accretion of neigboring smaller objects. Numerical simulations (Cohn 2005) predict higher cluster merger rates with increasing redshift, where the fraction of clusters with recent mass accretion due to mergers can be doubled at $z=0.7$, with respect to the local abundance. This framework provides a possible mechanism for preventing the formation of prominent cool cores at high redshift. However, since high- $z$ clusters are younger, other mechanisms may cause a delay in the formation of cool cores due to some internal energy release, such as AGN activity and star formation processes. The observed absence of pronounced $\mathrm{CC}$ at $z \geq 0.7$ is therefore plausible in the current cosmological framework.

We also investigated possible sample selection effects in the search for high redshift cool cores by studying 11 opticallyselected clusters from the RCS sample, which have a median temperature of $5 \mathrm{keV}$. We found that, unlike the X-ray sample, the majority of clusters in the RCS subsample have $c_{\mathrm{SB}}<0.075$, i.e., they lie in the non-cool core category.

To further extend this analysis we need to construct a complete low redshift sample, possibly with the same selection function as the distant sample, to be able to trace the evolution of the abundance of cool cores. A good prospect for this study is the local representative cluster sample XMM-Large Programme (Böhringer et al. 2007), which comprises 33 clusters in the redshift range $z=0.055$ to 0.183 , drawn from the REFLEX sample (Böhringer et al. 2001). An intermediate redshift sample $(z=0.3-0.6)$ from a similar project is also available (P. I. M. Arnaud), which provides an intermediate step in the assessment of the cool core evolution with redshift.

Clearly, having a high- $z$ data set covering a larger volume would also be very advantageous. Serendipitous cluster surveys currently underway, such as the XCS (Romer et al. 2001), the XDCP (Mullis et al. 2005; Böhringer et al. 2005) and XMM-LSS (Pierre et al. 2006), will yield suitable distant samples for these studies in the near future.

A comparison of our results with cosmological simulations would also provide additional new constraints to cluster formation scenarios. In fact, new insights on the evolution of simulated CCs and NCCs have recently been published in Burns et al. (2008), which are in good agreement with our findings. 
Acknowledgements. We would like to thank A. Baldi for giving access to the low redshift Chandra data. J.S.S. thanks Y.-Y. Zhang, G. Pratt, D. Pierini and I. Balestra for useful discussions. J.S.S. is supported by the Deutsche Forschungsgemeinschaft under contract BO702/16-2. P.T. and S.E. acknowledge the financial contribution from contract ASI-INAF I/088/06/0.

\section{References}

Allen, S. W., Ettori, S., \& Fabian, A. C. 2001, MNRAS, 324, 877

Arabadjis, J. S., Bautz, M. W., \& Garmire, G. P. 2002, ApJ, 572, 66

Arnaud, K. A. 1996, Astronomical Data Analysis Software and Systems V, ASP Conf. Ser., 101, 5, 17

Arnaud, M. 2006, A\&A, 454, L131

Arnaud, M., \& Evrard, G. 1999, A\&A, 86, 286

Arnaud, M., Aghanim, N., \& Neumann, D. M. 2002, A\&A, 389, 1

Arnaud, M., Pointecouteau, E., \& Pratt, G. W. 2005, A\&A, 441, 893

Baldi, A., Ettori, S., Mazzotta, P., Tozzi, P., \& Borgani, S. 2007, ApJ, 666, 835

Balestra, I., Tozzi, P., Ettori, S., et al. 2007, A\&A, 462, 429

Bauer, F. E., Fabian, A. C., Sanders, J. S., Allen, S. W., \& Johnstone, R. M. 2005, MNRAS, 359, 1481

Bignamini, A., Tozzi, P., Borgani, S., Ettori, S., Rosati, P., submitted

Böhringer, H., Voges, W., Huchra, J. P., et al. 2000, ApJ, 129, 435

Böhringer, H., Guzzo, L., Collins, C. A., et al. 2001, A\&A, 369, 826

Böhringer, H., Mullis, C. R., Rosati, P., et al. 2005, ESO Messenger, 120, 33

Böhringer, H., Schuecker, P., Pratt, G. W., et al. 2007, A\&A, 469, 363

Bouwens, R., Broadhurst, T., \& Silk, J. 1998, ApJ, 506, 557

Brueggen, M., \& Kaiser, C. R. 2002 Nature, 418, 301

Burns, J. O. 1990, AJ, 99, 14

Burns, J. O., Hallman, E. J., Gantner, B., Motl, P. M., \& Norman, M. L. 2008, ApJ, 675, 1125

Carroll, S. M., Press, W. H., \& Turner, E. L. 1992, ARA\&A, 30, 499

Cavaliere, A., \& Fusco-Femiano, R. 1976, A\&A, 49, 137

Chen, Y., Reiprich, T. H., Boehringer, H., Ikebe, Y., \& Zhang, Y.-Y. 2007, A\&A, 466, 805

Churazov, E., Forman, W., Jones, C., \& Bhringer, H. 2000, A\&A, 356, 788

Cohn, J. D., \& White, M. 2005, Astropart. Phys. 24, 316

De Filippis, E., Sereno, M., Bautz, M. W., \& Longo, G. 2005, ApJ, 625, 108

Edge, A. C., \& Freyer, D. T. 2003, ApJ, 594, 13

Eilek, J. A. 2003, Proceedings of The Riddle of Cooling Flows in Galaxies and Clusters of Galaxies, ed. T. Reiprich, J. Kempner, \& N. Soker [arXiv: astro-ph/0310011]

Ettori, S., Tozzi, P., Borgani, S., \& Rosati, P. 2004, A\&A, 188, 55

Evrard, A., Metzler, P., \& Navarro, J. 1996, ApJ, 469, 494

Fabian, A. C., Nulsen, P. E. J., \& Canizares, C. R. 1984, Nature, 310, 733

Fabian, A. C., Crawford, C. S., \& Mushotzky, R. F. 1994, MNRAS, 267, 779
Gladders, M., \& Yee, H. 2005, PASP, 201, 459

Hashimoto, Y., Boehringer, H., Henry, J. P., Hasinger, G., \& Szokoly, G. 2007, A\&A, 467, 485

Hicks, A., Ellingson, E., Bautz, et al. 2007 [arXiv: astro-ph/07105513]

Hogg, D., Baldry, I., Blanton, M., \& Eisenstein, D. 2002 [arXiv: astro-ph/0210394]

Jones, C., \& Forman, W. 1984, ApJ, 276, 38

Kaastra, J. S. 1992 (Internal SRON-Leiden Report, updated version 2.0)

Liang, H., Lmonon, L., Valtchanov, I., Pierre, M., \& Soucail, G. 2000, A\&A, 363,440

Markevitch, M. 2005 [arXiv:astro-ph/0511345]

Markevitch, M., \& Vikhlinin, A. 2001, ApJ, 563, 95

Martini, P., Kelson, D. D., Mulchaey, J. S., \& Athey, A. 2004, Proceedings of Clusters of Galaxies: Probes of Cosmological Structure and Galaxy Evolution, ed. J. S. Mulchaey, A. Dressler, \& A. Oemler

Mullis, C. R., Rosati, P., Lamer, G., et al. 2005, ApJ, 623, 85

Navarro, J. F., Frenk, C. S., \& White, S. D. M. 1996, ApJ, 462, 563

Navarro, J. F., Frenk, C. S., \& White, S. D. M. 1997, ApJ, 490, 493

Neumann, D. M., \& Arnaud, M. 1999, A\&A, 348, 711

O’Hara, T. B., Mohr, J. J., Bialek, J. J., \& Evrard, A. 2006, ApJ, 639, 64

Ota, N., \& Mitsuda, K. 2004, A\&A, 428, 727

Peres, C. B., Fabian, A. C., Edge, A. C., et al. 1998, MNRAS, 298, 416

Perlman, E. S., Horner, D. J., Jones, L. R., et al. 2002, ApJ, 140, 265

Perlmutter, S., and The Supernova Cosmology Project 1997, ApJ, 483, 565

Peterson, J. R., \& Fabian, A. C. 2005, Phys. Rep., 427, 1

Peterson, J. R., Kahn, S. M., Paerels, F. B. S., et al. 2003, ApJ, 590, 207

Pierre, M., Pacaud, F., Duc, P.-A., et al. 2006, MNRAS, 372, 591

Pratt, G. W., \& Arnaud, M. 2002, A\&A, 394, 375

Poole, G. B., Fardal, M. A., Babul, A., et al. 2006, MNRAS, 373, 881

Reese, E. D., Carlstrom, J. E., Joy, M., et al. 2002, ApJ, 581, 53

Rosati, P., Della Ceca, R., Norman, C., \& Giacconi, R. 1998, ApJ, 492, 21

Rosati, P., Stanford, S. A., Eisenhardt, P. R., et al. 1999, AJ, 180, 76

Romer, A. K., Viana, P. T. P., Liddle, A. R., \& Mann, R. G. 2001, ApJ, 547, 594

Sanderson, A. J. R., Ponman, T. J., \& O’Sullivan, E. 2006, MNRAS, 372, 1496

Sarazin, C. 1988 (Cambridge University Press)

Stanford, S. A., Holden, B., Rosati, P., et al. 2002, AJ, 123, 619

Stanford, S. A., Romer, A. K., Sabirli, K., et al. 2006, ApJ, 646, 13

Vikhlinin, A., Van Speybroeck, L., Markevitch, M., Forman, W. R., \& Grego, L. 2002, ApJ, 578, 107

Vikhlinin, A., Markevitch, M., Murray, S. S., et al. 2005, ApJ, 638, 655

Vikhlinin, A., Burenin, R., Forman, W. R., et al. 2006, Proceedings of Heating vs. Cooling in Galaxies and Clusters of Galaxies, ESO Astrophysics Symposia, ed. H. Böhringer, G. W. Pratt, A. Finoguenov, \& P. Shuecker [arXiv: astro-ph/0611438]

Zhang, Y.-Y., Finoguenov, A., Böhringer, H., et al. 2006, A\&A, 86, 286 